\title{
The Effectiveness of Teaching Non-Native Learners in Recognizing the Differences of 4 Tones using a Romanised Mandarin Web-based Instruction
}

\author{
Lim Soo Giap*, Ong Sheau Fen \\ Academy of Language Studies, Universiti Teknologi MARA (UiTM), Malaysia \\ *limsoogiap266@ppinang.uitm.edu.my
}

\begin{abstract}
The objective of this paper is to assess the feasibility of utilizing Guru Hanyu Pinyin web based instruction as a teaching tool for non-native speakers in recognizing the differences of 4 tones at syllable level in Hanyu Pinyin. The study compares the academic achievements for Hanyu Pinyin of two different groups of students whereby the experimental group adopts the web based instruction and the control group adopts the traditional approach as the mode of learning. This research is targetted on first year elementary level Mandarin students at UiTM Penang campus. The research sample has been selected through stratified random sampling. Research findings reveal interesting results whereby the performance of the control group is statistically better than the experimental group in recognizing differences of 4 tones at syllable level. This paper also discusses some limitations that were discovered in the existing version of teaching tool developed and proposes some recommendations for future research.
\end{abstract}

\section{Keywords: Romanised Mandarin; web-based instruction; recognizing 4 tones.}

\section{Introduction}

Hanyu Pinyin (Romanised Mandarin) is used in the teaching and learning of Mandarin as a third language in UiTM (University of Technology of MARA). Generally, the first 2 to 4 hours of lecture are allocated to the teaching of the sound system in Hanyu Pinyin. The time allocated is definitely insufficient for learners, many of whom are exposed to Mandarin for the first time, to satisfactorily comprehend the intricacies of its sound system. In order to achieve better learning efficiency, there is a strong need for lecturer to spend extra hours with the learners onr additional practice and consultations. Hence, it is necessary to devise some self-learning materials for learners to enable them to control and monitor their learning process in the absence of facilitator. In order to create an environment conducive for self-learning Hanyu Pinyin, a web-based instruction is suggested to be incorporated into the curriculum.

Web-based instruction, which is also called as Web-based training, is defined as an "individualised instruction that is delivered over public or private computer networks and displayed by a Web browser". WBT is not a downloaded Computer Based Training but an on-demand training stored in a server and accessed across a network. Web-based training can be updated very rapidly and its accessibility as a training tool is fully controlled by the training provider (Clark, 1996). It generally applies to any kind of instructional materials delivered over the Internet accessed by browser-equipped computer users. However, before such a measure can be taken, it is important to investigate its effectiveness in enhancing the learning of Hanyu Pinyin.

The Guru Hanyu Pinyin web-based instruction ${ }^{1}$ is an internet enabled web-based instruction specially designed by a group of UiTM lecturers to overcome the weaknesses in learning Mandarin pronunciation based on the specific needs of Bumiputra learners (non-native learners). It is designed on the basis of instructional system design (ISD) methodology. This approach involves four phases: (1) analysis and design, (2) development, (3) implementation, and (4) evaluation of the instruction. For tone pronunciation errors, the researchers found that there are mainly 4 categories of errors. The errors are pronouncing the first tone, second tone, third tone and fourth tone incorrectly. Lee, et al. (2004) found that the sequences of pronunciation error from highest to lowest are the second tone (59.5\%); the fourth tone $(52.8 \%)$, the third tone $(36.7 \%)$ and the first tone $(27.5 \%)$. In other words, averagely, learners make most mistakes in pronouncing second tone, followed by the fourth tone, subsequently the third tone and lastly the first tone . 
The objective of this study is to determine the suitability of utilizing Guru Hanyu Pinyin web based instruction as a teaching toolin teaching Hanyu Pinyin for non-native speakers in recognizing differences of 4 tones at syllable level. The study intends to compare the academic achievements for Hanyu Pinyin between the students who study through web based instruction and those who study through the traditional approach. Both research question and statistical comparison mode are well described:

Is the difference between the means of recognizing differences of 4 tones at syllable level between experimental group and control group statistically significant? To compare the academic achievements of learners who learn through the web based instruction and those who learn through the traditional approach, a t- test is employed : choose the correct tone after listening to a syllable given, the purpose of this test is to examine students' listening skills at syllable level.

The hypotheses are written as below to answer research question:

$\mathrm{H}_{1}$ : There is significant difference between the means of recognizing differences of 4 tones at syllable level between experimental group and control group.

and the null hypotheses is

$\mathrm{H}_{0}$ : There is no significant difference between the means of recognizing differences of 4 tones at syllable level between experimental group and control group.

\section{Literature Review}

The key elements used in this research are UiTM, Hanyu Pinyin, WBI (Web-based instruction), Mandarin facilitator, non native Mandarin learners, Chinese language learning on the Internet, and the Hanyu Pinyin WBI on the Internet.

UiTM: UiTM is University of Technology of MARA. The university is selected for this research in view of its large population of Bumiputra students from various disciplines. In order to equip their students with language skills, UiTM also offer several of foreign language courses including Mandarin to the students.

Hanyu Pinyin: Hanyu Pinyin is a form of Romanized Mandarin. It was devised by the government of the Peoples' Republic of China in 1958 to help non-native speakers to study Chinese. It is the most widely used Mandarin transliteration method which has been adopted in many countries including Singapore. Most contemporary dictionaries use Pinyin to spell out Chinese characters, and the vast majority of Chinese textbooks published overseas use Pinyin (Zhang, 2006). By mastering Pinyin, learners can easily read Chinese books printed in characters which are at the same time transliterated into Pinyin.

Web Based Instruction (WBI): Khan (1997) defines Web-Based Instruction (WBI) as “...a hypermediabased instructional program which utilizes the attributes and resources of the World Wide Web to create a meaningful learning environment where learning is fostered and supported." According to Relan and Gillami (1997) WBI is "...the application of a repertoire of cognitively oriented instructional strategies within a constructivist and collaborative learning environment, utilizing the attributes and resources of the World Wide Web." Web-Based Instruction, also called Web-Based Training, is defined by Clark (1996) as an "Individualized instruction delivered over public or private computer networks and displayed by a Web browser. WBT is not downloaded CBT, but rather on-demand training stored in a server and accessed across a network. Web-based training can be updated very rapidly, and its accessibility as a training tool is controlled by the training provider." Clearly, as the name suggests, one common feature for all forms of WBI is that the instructional materials are delivered over the Internet.

Non Native Mandarin Learner: Non native refers to the people whose mother tongue is not Mandarin. Non-native Mandarin learners are Bumiputras that speak Malay language as mother tongue. Learner is someone who learns to speak Mandarin. Non native learner is the Bumiputra who learns to speak Mandarin.

UiTM Mandarin Lecturer / Facilitator: Lecturer / Facilitator is someone who helps a group of people discuss things with each other or do something effectively (Longman Dictionary of Contemporary, 1995). In UiTM, the Mandarin lecturer plays the role as a facilitator who helps the non native learners to study Mandarin effectively. 
Chinese Language learning on the Internet: According to Bourgerie (2003), the Internet sources for learning Chinese can be as simple as supplements for established texts or comprehensive courses such as Beijing Language and Culture University's on-line degree program for Chinese. Other on-line sources consist of independent modules to address a particular aspect of learning such as pronunciation and Romanization Hanyu Pinyin WBI on the Internet. Among the well-designed and established Hanyu Pinyin WBI according to Shao (2005), are the Chinese Pronunciation Guide of Harvard University and the Pinyin Pronunciation for Mandarin of Oxford. The contents of these two websites are not designed and arranged in accordance with any existing teaching materials and as a result they can be modified easily. Similarly, they can be accessed by not only students from the two universities but the public as well. Since the two sites are not based on existing teaching materials, they focus mostly on vowels, consonants, word-level and phrase-level tones. For learners, they benefit from these two sites more on the learning of word-level rather than sentence-level tones. In terms of feedback for learners, there is no such provision on both sites.

\section{Research Design and Methodology}

Sample and Sampling Techniques: This research focuses on first year elementary level Mandarin students at UiTM Penang campus. The research sample has been selected through stratified random sampling. They represent the Bumiputra Mandarin learners and the majority of Bumiputra learners have similar education background, culture, and language ability. Furthermore, the focus on UiTM Penang students is to make ease for research accessing and monitoring.

Data Collection: Total 28 students in UiTM Penang campus were selected to attend a 4-hour self-learning session using Hanyu Pinyin instruction at the language computer laboratory, another 28 students act as the control group who were taught using the conventional teaching approach with no exposure to Hanyu Pinyin web-based instruction. A Hanyu Pinyin test results is used to collect data for the research question. However, for the control group, only 26 students turned up for the test.

Techniques for Analysis: The result of achievement test of the experimental group (the group that adopted the web based instruction in learning Hanyu Pinyin) and the control group (the group that adopted the traditional approach) are tabulated and coded in the data file for the use of SPSS statistical analysis. The achievement test is analyzed using the means, standard deviations and Independent Samples t-test of the SPSS package to compare the means of the two groups.

\section{Results and Discussion}

Table 1: Independent Sample t-test

\begin{tabular}{llllllcc}
\multicolumn{1}{c}{ Group } & N & F & Sig. & Mean & SD & $\begin{array}{c}\text { t-value } \\
\text { (2-tailed) }\end{array}$ & $\begin{array}{c}\text { Sig(2- } \\
\text { tailed) }\end{array}$ \\
\hline $\begin{array}{l}\text { Experimental Group } \\
\text { Control Group }\end{array}$ & 28 & \multirow{2}{*}{6.355} & 0.015 & 7.3571 & 2.31284 & -2.363 & 0.022 \\
\hline
\end{tabular}

As shown in Table 1, the means score of the experimental group and the control groups are 7.3571 and 8.6154 respectively. The results also shows Levene's Test for equality of variance $F_{0.95,52}=6.355, P>0.05$, we assume the variances are not equal.With refer to t-test for equality of means, the null hypothesis that there is no significant difference between the means of recognizing differences of 4 tones at syllable level between experimental group and control group is rejected since $p$-value $=0.022<0.05$. Therefore, we conclude that there is significant difference between the means of recognizing differences of 4 tones at syllable level between experimental group and control group.

\section{Conclusions}

The $p$-value $=0.015$, of equal variances not assumed $(\mathrm{p}<0.05)$ (refer to Table 1 ) indicates that there is significant statistical difference between score of the control group and the experimental group in recognizing differences of 4 tones at syllable level. So the $\mathrm{H}_{0}$ is rejected. The means score of control groups is higher than the experimental group indicated that the performance of control group is better than the experimental group. 4 tones in Mandarin are 4 different tonal pitches in pronunciation. Though they are not many, the sound differences are quite close and pose a challenge for non native speakers to 
accurately recognize them. Lecturer needs to use a very special and exaggerate teaching approach to enhance the understanding and deepen the memory of students in recognizing them. One of the main reasons for the control group to perform better in differentiating 4 tones than the experimental group is the content of web based instruction use in this research is lack of this special expression-mode of teaching approach compare to the on-campus lecturer. Therefore, it is proven that the presence of the lecturer in teaching and learning process of 4 tones is important whereas the web-based instruction is more appropriate to be used as a supplementary tool in learning process and as a self-learning tool in the absence of lecturer.

The other reason that explains these interesting findings is the limitations of WBI itself could be the main cause of having control group outperformed control group in differentiating 4 tones. In terms of pedagogy, web-based learning does not have the advantage of promoting spontaneous interaction as in face to face communication. Traditional approach using on-campus learning focuses students' learning attention through easy communication with lecturer whereby lecturer could offer help to explain in more detail and provide interactive feedback about students' accuracy in pronouncing the 4 tones. Furthermore, WBI does not tailored to individual student's specific needs, specifically in offering opportunities to practice speaking, listening and writing skills to master the learning of 4 tones. Thus, WBI is more suitable to be used in supplementing the traditional on-campus approach of teaching Hanyu Pinyin. Learners should first gain the basics from classroom teaching prior to using this supplementary tool. Learners have to exert more mental effort and spend more self-learning time if they solely depend on WBI without any guidance from lecturer.

\section{Limitations and Recommendations}

Poorly equipped WBI users (learners) on technical skills such as installing plug-in software, volume control and etc. is reflected in poor learning effectiveness of 4 tones in Hanyu Pinyin study. It is recommended that the users are being provided with some technical training prior to using WBI. Without adequate technical skills equipped, the learners might not be able to use the web-based instruction effectively. The WBI used is lack of a suitable infrastructure and database for accommodating test item and multimedia elements. Therefore it is recommended that the database should be attached to the instruction in order to allow adding, deleting and modifications of exercises and quiz items being carried out with more flexibility. The existing WBI design requires further improvements to encompass a well structured and interesting interface design with course syllabus and content. This will enable the web-based learning technology being easily accepted by new learners in view of its convenience and user-friendlyness. Despite the discussions above, the contents of the syllabus should also be revised as most syllabi in the WBI used in this research are designed for conventional classroom learning environment.

Colourful websites with more sounds and animations with downaloadable audio and video lessons, coupled with interesting quizzes and games should be incorporated to suit web-based learning. With this, the web-based learning becomes more interactive and less passive and thus, able to attract students to learn 4 tones with minimal dependency on the presence of lecturer. It is recommended that the future research could target on the areas described for web based course module development. Besides that, replication of the study can be carried out in primary school and secondary school to investigate the effectiveness of WBI in enhancing the learning of Hanyu Pinyin. In short, WBI would still play a major role in improving the effectiveness of 4 tones Hanyu Pinyin learning once the above limitations discussed are being resolved as compared to the conventional classroom learning approach in the near future. 


\section{References}

Bourgerie, D. S. (2003). Computer Aided Language Learning for Chinese: A Survey and Annotated Bibliography. Journal of the Chinese Language Teachers Association, 38(2), 17-47.

Clark, G. (1996). Glossary of CBT/WBT Terms. Retrieved January 07, 2010 from http://www.clark.net/pub/nractive/alt5.htm.

Khan, B. H. , (1997). (Ed.) Web-Based Instruction, 1997, Educational Technology Publications, Englewood Cliffs, New Jersey: 6.

Lee, A. C. , Lau, S. K. \& Mok , S. S. (2004). Kesilapan sebutan fonem awal dan nada dalam pertuturan bahasa mandarin : satu kajian kes terhadap pelajar intersesi peringkat Diploma, UiTM Shah Alam / Lee Ai Chat , Lau Suk Khin, Mok Soon Sim. Technical Report. Institute of Research, Development and Commercialization, Universiti Teknologi MARA.

Longman Dictionary of Contemporary English. (1995). Harlow:Longman, Third Edition.

Relan, A. \& Gillani, B. B. (1997). Web-Based Information and the Traditional Classroom: Similarities and Differences. In Khan, B.H., (Ed.), Web-Based Instruction, 1997a, Educational Technology Publications, Englewood Cliffs, New Jersey: 43.

Shao, B. (2005). A brief introduction of Online Hanyu Pinyin Web Sites] [Online].Taiwan: International Conference on Internet Chinese Education. Retrieved December 20, 2010 from http://edu.ocac.gov.tw/discuss/academy/netedu04/ICICE2005/html/paper2/C12.pdf

Zhang, J. (2006). Hanyu Pinyin for Mandarin Speakers, [Online]. Boston:MIT. Retrieved November 21, 2010 from http://web.mit.edu/ jinzhang/www/pinyin/ 